\title{
A novel prognostic signature for idiopathic pulmonary fibrosis based on five-immune-related genes
}

\author{
Lingxiao Qiu ${ }^{1,2,3} \wedge$, Gencheng Gong ${ }^{4}$, Wenjuan $\mathrm{Wu}^{5}$, Nana $\mathrm{Li}^{1} \wedge$, Zhaonan $\mathrm{Li}^{6}{ }^{\circ}$, Shanshan Chen ${ }^{1,3}$, Ping $\mathrm{Li}^{1,3,7}$, \\ Tengfei Chen ${ }^{1}$, Huasi Zhao ${ }^{1}$, Chunling Hu ${ }^{8}$, Zeming Fang ${ }^{9}$, Yan Wang ${ }^{1}$, Hongping Liu ${ }^{1}$, Panpan Cui ${ }^{10}{ }^{\wedge}$, \\ Guojun Zhang ${ }^{1,3,7} \wedge$
}

${ }^{1}$ Department of Respiratory Medicine, The First Affiliated Hospital of Zhengzhou University, Zhengzhou, China; ${ }^{2}$ Academy of Medical Sciences, Zhengzhou University, Zhengzhou, China; ${ }^{3}$ Henan Provincial Medical Key Laboratory for Interstitial Lung Disease and Lung Transplantation, Zhengzhou, China; ${ }^{4}$ State Key Laboratory of Respiratory Disease, National Clinical Research Center for Respiratory Disease, Guangzhou Institute of Respiratory Health, the First Affiliated Hospital of Guangzhou Medical University, Guangzhou, China; ${ }^{5}$ Department of Geriatric Medicine, Henan Provincial People's Hospital, Zhengzhou, China; ${ }^{6}$ Department of Interventional Radiology, The First Affiliated Hospital of Zhengzhou University, Zhengzhou, China; ${ }^{7}$ Zhengzhou Key Laboratory for Chronic Respiratory Disease, Zhengzhou, China; ${ }^{8}$ Department of Respiratory Intensive Care Unit, The First Affiliated Hospital of Zhengzhou University, Zhengzhou, China; ${ }^{9}$ Department of Thoracic Surgery, The First Affiliated Hospital of Zhengzhou University, Zhengzhou, China; ${ }^{10}$ School of Nursing and Heath, Zhengzhou University, Zhengzhou, China

Contributions: (I) Conception and design: L Qiu, G Gong; (II) Administrative support: G Zhang; (III) Provision of study materials or patients: L Qiu, C Hu; (IV) Collection and assembly of data: L Qiu, W Wu, Z Li; (V) Data analysis and interpretation: L Qiu, G Gong, N Li, P Cui; (VI) Manuscript writing: All authors; (VII) Final approval of the manuscript: All authors.

Correspondence to: Guojun Zhang. The First Affiliated Hospital of Zhengzhou University, No. 1 Jianshe East Road, Zhengzhou 450000, China. Email: zgj@zzu.edu.cn.

Background: Idiopathic pulmonary fibrosis (IPF) is a highly fatal lung disease of unknown etiology with a median survival after diagnosis of only 2-3 years. Its poor prognosis is due to the limited therapy options available as well as the lack of effective prognostic indicators. This study aimed to construct a novel prognostic signature for IPF to assist in the personalized management of IPF patients during treatment.

Methods: Differentially-expressed genes (DEGs) in IPF patients versus healthy individuals were analyzed using the "limma" package of R software. Immune-related genes (IRGs) were obtained from the ImmPort database. Univariate Cox regression analysis was adopted to screen significantly prognostic IRGs for IPF patients. Multiple Cox regression analysis was used to identify optimal prognostic IRGs and construct a prognostic signature.

Results: Compared with healthy individuals, there were a total of 52 prognosis-related DEGs in the bronchoalveolar lavage (BAL) samples of IPF patients, of which 37 genes were identified as IRGs. Of these, five genes (CXCL14, SLC4OA1, RNASE3, CCR3, and RORA) were significantly associated with overall survival (OS) in IPF patients, and were utilized for establishment of the prognostic signature. IPF patients were divided into high- and low-risk groups based on the prognostic signature. Marked differences in the OS probability were observed between high- and low-risk IPF patients. The area under curves (AUCs) of the receiver operating characteristic (ROC) curve for the prognostic signature in the training and validation cohorts were 0.858 and 0.837 , respectively. The expression levels between RNASE3 and SLC40A1 ( $\mathrm{P}<0.01$, $\mathrm{r}=0.394)$, between RORA and CXCL14 ( $\mathrm{P}<0.01, \mathrm{r}=-0.355)$, between CCR3 and CXCL14 $(\mathrm{P}<0.01, \mathrm{r}=0.258)$, as well as between RNASE3 and CCR3 $(\mathrm{P}<0.01, \mathrm{r}=0.293)$ were significantly correlated.

Conclusions: We developed a validated and reproducible IRG-based prognostic signature that should be helpful in the personalized management of patients with IPF, providing new insights into the relationship between the immune system and IPF.

\footnotetext{
^ ORCID: Lingxiao Qiu, 0000-0002-1703-0797; Nana Li, 0000-0003-2882-7045; Zhaonan Li, 0000-0003-3055-8817; Panpan Cui, 00000002-1587-7863; Guojun Zhang, 0000-0003-1756-0082.
} 
Keywords: Idiopathic pulmonary fibrosis (IPF); prognostic signature; bronchoalveolar lavage (BAL); immune-related genes (IRGs); GEO

Submitted Aug 31, 2021. Accepted for publication Oct 02, 2021.

doi: $10.21037 / \mathrm{atm}-21-4545$

View this article at: https://dx.doi.org/10.21037/atm-21-4545

\section{Introduction}

Idiopathic pulmonary fibrosis (IPF) is a deadly interstitial lung disorder of unknown etiology (1). It is characterized by irreversible fibrogenesis in the lung parenchyma, leading to progressive respiratory function failure and eventually death $(2,3)$. IPF is the most common interstitial lung disease and has the worst prognosis in pulmonary fibrosis (4). Nearly half of IPF patients die within 2-3 years after diagnosis $(3,4)$, and the 5 -year survival rate is less than $30 \%(5)$. IPF is a highly heterogeneous disease with a greatly variable natural history $(6,7)$. The course of this disease in an individual patient is difficult to predict $(4,8)$; some patients with IPF experience rapid decline, while others experience much slower development $(3,8)$. For a long time, the lack of effective prognostic indicators has made it difficult to accurately track and evaluate the prognosis of IPF, which has led to the poor prognosis of IPF to a certain extent. Hence, the development of applicable prognostic signatures is urgently needed for the clinical treatment of IPF.

The pathophysiological pathogenesis of IPF involves aberrant transcription and gene expression (9-14). Molecular genomic features based on lung tissue have been used to predict the development of IPF $(15,16)$. Though previous studies have identified some genes and pathways may play an important role in the occurrence and development of IPF, and may be expected to be biomarkers or therapeutic targets for the diagnosis of IPF $(17,18)$. However, the lack of verification of survival information is the biggest short board in these papers. Meanwhile, the resources required to perform a lung biopsy and the risks associated with the procedure limit the applicability of such genomic features. Molecular models have also been established based on peripheral blood mononuclear cell (PBMC) transcription profile data to predict the disease state of IPF $(19,20)$. However, in the absence of lung biopsies, it is difficult to explain the correlation between abnormal PBMC transcription and pulmonary fibrosis course. Bronchoalveolar lavage (BAL) is a method of obtaining alveolar surface lining fluid with fiberoptic bronchoscopy for evaluating inflammation, immune cells, and soluble substances. BAL plays a vital role in assisting IPF diagnosis and has been recommended as the auxiliary diagnostic reference by the American Thoracic Society (ATS) (21). The advantages of utilizing the gene expression profiles of BAL cells to depict the molecular features of IPF include lung localization, ease of accessibility, and dynamic assessment of disease status through longitudinal sample collection. Previous studies have revealed that Innate and adaptive immune responses disorders possess an important role in the pathogenesis of lung fibrosis (22). The differentially-expressed immune-related genes (IRGs) also have been reported associated with the development of IPF $(23,24)$. The immPort database is funded by the National Institutes of Health (NIH), National Institute of Allergy and Infectious Diseases (NIAID), Health and Human Services (HHS) in support of the NIH mission to share data with the public. It provides information about the immunerelated genes of humans. Therefore, using the GSE70866 gene expression data set of the Gene Expression Synthesis (GEO) database and the IRGs list of the ImmPort database, we aim to combine the survival information of IPF patients to establish a new molecular genome feature screening from IRGs, to predict the prognosis of IPF patient. We present the following article in accordance with the STARD reporting checklist (available at https://dx.doi.org/10.21037/ atm-21-4545).

\section{Methods}

\section{Acquisition and analysis of datasets}

Microarray profile data from the GSE70866 gene expression dataset were downloaded from the GEO (http://www.ncbi. nlm.nih.gov/geo/) database. The platform was a GPL14550 Agilent-028004 SurePrint G3 Human GE 8x60K Microarray (Agilent Technologies Inc., California, U.S.). A total of 132 BALF samples, including 20 samples from healthy individuals and 112 samples from IPF patients, were used to analyze the microarray data. All 112 IPF patients had detailed sociodemographic characteristics and complete survival information. The study was conducted in accordance with 
the Declaration of Helsinki (as revised in 2013).

\section{The criteria of differentially-expressed genes (DEGs) and differentially-expressed immune-related genes (IRGs)}

The filtration of DEGs was performed in 112 IPF patients versus healthy individuals. In this study, DEGs between IPF and healthy individuals were defined using a $\log 2$ fold change (FC) $>1$ and an adjusted $\mathrm{P}$ value (adj. $\mathrm{P}$ ) $<0.05$ as thresholds. A total of 1,793 IRGs were obtained from the ImmPort (https://www.immport.org/shared/genelists) database. Taking the intersection through the Venn algorithm (http://bioinformatics.psb.ugent.be/webtools/ Venn/), 52 differentially-expressed IRGs were filtered, which remained and were used as candidates for subsequent analysis.

\section{Construction and validation of the prognostic IRG-based signature}

The 112 included patients were randomly divided into a training cohort $(50 \%)$ and validation cohort $(50 \%)$ using the random numbers method. The construction of prognostic gene-based signatures was carried out in the training cohort, and verification was performed in the verification cohort. Univariate Cox regression analysis was used to screen for immune genes that were significantly associated with prognosis, with a cut-off of $\mathrm{P}<0.05$. Next, multivariate Cox-regression analysis was performed on the training cohort to further determine the best prognostic IRG signature using the "survival" package (URL: https:// github.com/therneau/survival) in $\mathrm{R}$ software (version 4.0.3) (URL: https://cran.r-project.org/mirrors.html), with a cutoff of $\mathrm{P}<0.05$. The formula of IPF patient's risk score was established as follows: score $=$ sum (each gene's expression $\times$ corresponding coefficient). The patients were stratified into high-risk and low-risk groups based on the median value of the risk score. Based on the risk score groups, survival differences between high-risk and low-risk groups were carried out with the "survival" R package (URL: https:// github.com/therneau/survival).

\section{Statistical analysis}

Baseline characteristics such as age, sex, race, days to death, and vital status were collected. Continuous variables were reported as the mean ( \pm standard deviation) and compared using the Student's $t$-test. Categorical variables were reported as counts $\mathrm{n}(\%)$ and compared using the chi-square test. The comparison of sociodemographic features between the training and validation cohorts was carried out using GraphPad Prism (version 7.0; GraphPad Software, La Jolla, CA, USA).

The other statistical analyses were carried out using R software (version 4.0.3) (URL: https://cran.r-project. org/mirrors.html) and considered significant when the corresponding $\mathrm{P}<0.05$. The adjusted $\mathrm{P}<0.05$ was used for screening DEGs, and $\mathrm{P}<0.05$ was used as a significance threshold in the remaining statistical analyses. The analysis of DEGs was conducted by utilizing the "limma" package (URL: http://www.strimmerlab.org/software/ st/). Univariate Cox regression analysis was used to screen for DEGs that were significantly associated with overall survival (OS). Multivariate Cox regression analysis was performed on the training cohort to further determine the best prognostic IRG model. A multivariate Cox regression model was conducted for the variables with $\mathrm{P}<0.05$ in the univariate analyses. A gene-based signature was built with the coefficients of each factor in the multivariate Cox analysis. The "survival" package (URL: https://github.com/ therneau/survival) calculated the survival curve function, and the "survminer" package (URL: https://mirror.lzu.edu. cn/CRAN/bin/windows/contrib/4.0/survminer_0.4.9.zip) executed the visualization. The heat map was drawn using the "pheatmap" (pretty heatmap) package (URL: https:// mirror.lzu.edu.cn/CRAN/bin/windows/contrib/4.0/ pheatmap_1.0.12.zip). The volcano map was drawn using the "ggplot2" package (URL: https://cran.r-project.org/ web/packages/ggplot2movies/index.html).

\section{Results}

\section{Baseline characteristics of patient with IPF}

Table 1 summarizes the sociodemographic information of the included IPF patients. A total of 112 IPF patients were identified, with a median age of $69.5( \pm 10.1)$ years. IPF was more common in older populations $(67.0 \%$ of patients were older than 65 years versus $33.0 \%$ of patients less than 6 years). The incidence of IPF was higher in men than in women (83.0\% male patients versus $17.0 \%$ female patients).

These 112 IPF patients were randomly divided into training $(50 \%)$ and validation $(50 \%)$ cohorts, with 56 patients in each group. No significant differences between the two cohorts were observed in terms of age, sex, days to death, and vital status $(P>0.05)$. Qualified 
Table 1 The sociodemographic information of patients

\begin{tabular}{|c|c|c|c|c|}
\hline Characteristics & Total $(n=112)$ & Training cohort $(n=56)$ & Validation cohort $(n=56)$ & $P$ value \\
\hline \multicolumn{5}{|l|}{ Age, n (\%) } \\
\hline$<65$ & $37(33.0)$ & $18(32.1)$ & $19(34.0)$ & \\
\hline$\geq 65$ & $75(67.0)$ & $38(67.9)$ & $37(66.0)$ & 0.841 \\
\hline Female & $19(17.0)$ & $7(12.5)$ & $12(21.4)$ & \\
\hline Male & $93(83.0)$ & $49(87.5)$ & $44(78.6)$ & 0.208 \\
\hline Days to death, mean $( \pm S D)$ & $698.1( \pm 555.9)$ & $656.7( \pm 551.9)$ & $739.5( \pm 561.7)$ & 0.433 \\
\hline \multicolumn{5}{|l|}{ Vital status, n (\%) } \\
\hline \multicolumn{5}{|l|}{ Sample contact country, n (\%) } \\
\hline Germany & $112(100.0)$ & $56(100.0)$ & $56(100.0)$ & NA \\
\hline
\end{tabular}

$\mathrm{SD}$, standard deviation.

survival information for all of the included IPF patients was available for further analysis.

\section{Identification of DEGs}

DEGs of the IPF and healthy individuals from the GPL14550 platform of the GSE70866 gene expression dataset were analysed using the "limma" package. In this dataset, a total of 379 DEGs met the criteria, of which 207 genes were upregulated and 172 genes were downregulated (Table S1). Figure $1 A$ is a volcano map of 379 DEGs in the IPF group compared to the healthy individuals group. The profiling of all the DEGs is shown in Figure $1 B$ and presented in the form of a non-cluster analysis expression heatmap. SPP1, PPBP, and MMP7 were the top three most significantly upregulated genes in the IPF group, while NALCN, C8B, and ITIH5 were the three most downregulated genes in the IPF group.

\section{Identification of differential expression IRGs}

Combining the results of DEGs (Table S1) and the IRGs from the ImmPort database, 52 differentially expressed IRGs were identified. A volcano map was constructed to present the differential expression of all IRGs (Figure 2A). Figure $2 B$ shows the expression of the 52 differential IRGs in the form of a heatmap. SPP1, PPBP, TUBB3, CCL2, and S100A12 were the five most significantly upregulated IRGs, while the top five downregulated IRGs were PTGER3, CD40LG, CAMP, IGF1, and CXCL9.

\section{Prognostically relevant IRGs filtration}

Prognostically relevant IRGs for IPF were selected based on the results of univariate Cox regression analysis. A forest plot was drawn to show the 37 obtained prognostically relevant IRGs, including prognostically protective IRGs such as RORA [hazard ratio (HR): $0.613,95 \%$ confidence interval (CI): (0.474-0.794)] and ICOS [HR: 0.672, 95\% CI: (0.560-0.809)] (Figure 3). Conversely, MPO [HR: 1.287, 95\% CI: (1.139-1.454)], RNASE3 [HR: 1.711, 95\% CI: (1.338-2.188)], PDGFA [HR: 1.228, 95\% CI: (1.030-1.465)], PPBP [HR: 1.154, 95\% CI: (1.002-1.330)], and FABP3 [HR: 1.522, 95\% CI: (1.216-1.905)] were prognostic factors of worse survival (Figure 3).

\section{An IRGs prognostic model of IPF}

Multivariate Cox regression analysis was performed based on 37 prognostic factors of OS to establish a model to predict the outcomes of IPF patients. CXCL14, SLC40A1, RNASE3, CCR3, and RORA were ultimately identified to 



Figure 1 Comparison of the gene expression profile between the IPF group and the healthy individuals group. (A) Heatmap of significantly DEGs. (B) Volcano map of DEGs; red dots represent upregulated DEGs, grey dots represent non-differentially expressed genes, and green dots represent downregulated DEGs. IPF, idiopathic pulmonary fibrosis; DEGs, differentially-expressed genes. 

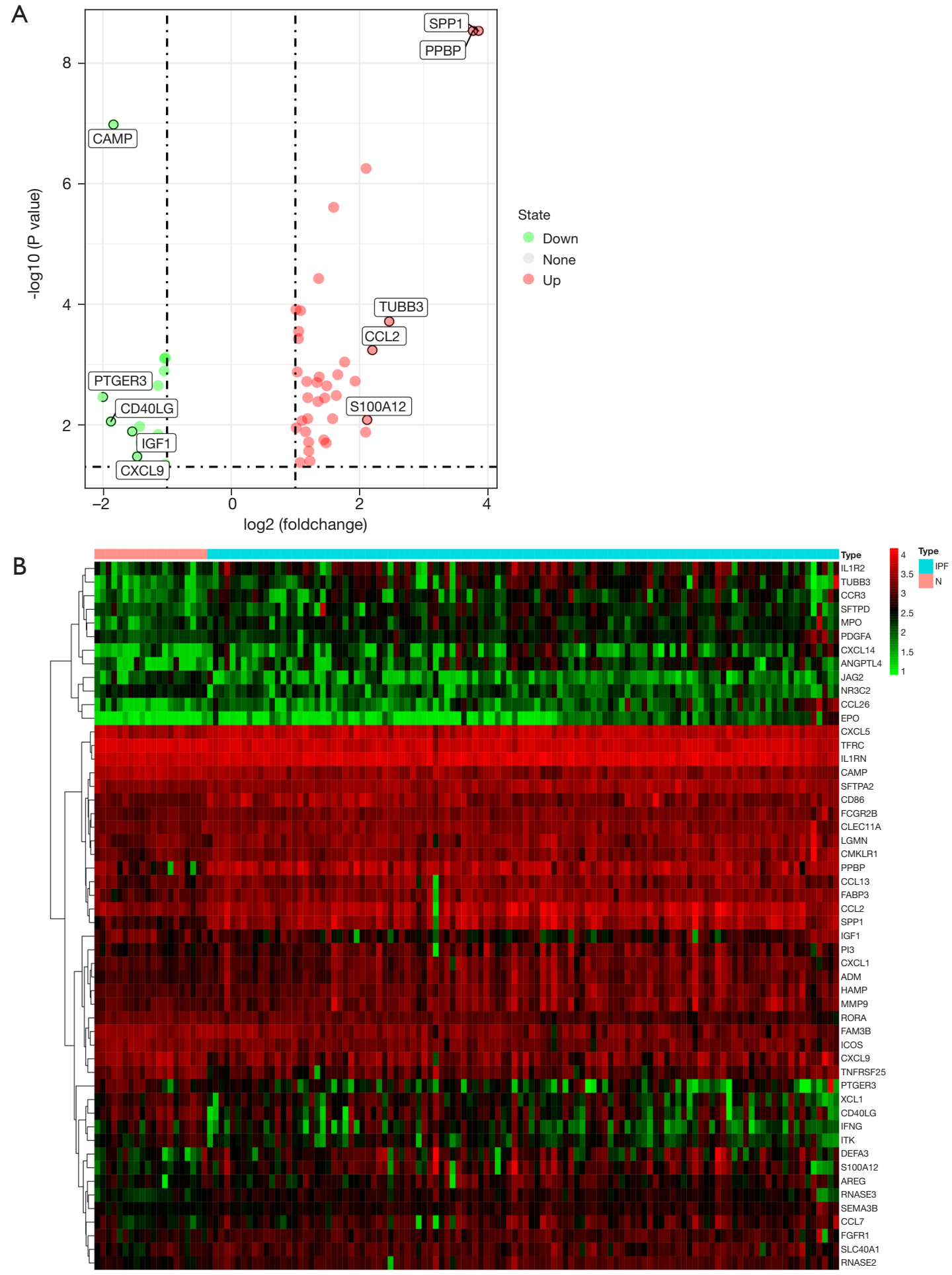

Figure 2 Comparison of the IRG expression profile between the IPF group and the healthy individuals group. (A) Heatmap of significantly differentially-expressed IRGs. (B) Volcano map of IRGs; red dots represent upregulated differentially expressed IRGs, grey dots represent non-differentially expressed IRGs, and green dots represent downregulated differentially expressed IRGs. IRG, immune-related gene; IPF, idiopathic pulmonary fibrosis. 


\begin{tabular}{|c|c|c|}
\hline $\begin{array}{l}\text { LGMN } \\
\text { HAMP } \\
\text { PI3 } \\
\text { CAMP } \\
\text { PPBP } \\
\text { CXCL14 } \\
\text { CXCL1 } \\
\text { CCL13 } \\
\text { DEFA3 } \\
\text { S100A12 } \\
\text { MMP9 } \\
\text { SLC40A1 } \\
\text { SFTPA2 } \\
\text { FABP3 } \\
\text { MPO } \\
\text { RNASE3 } \\
\text { CCL26 } \\
\text { CCR3 } \\
\text { CCL2 } \\
\text { CCL7 } \\
\text { RNASE2 } \\
\text { FCGR2B } \\
\text { SEMA3B } \\
\text { CMKLR1 } \\
\text { ADM } \\
\text { AREG } \\
\text { FAM3B } \\
\text { IL1RN } \\
\text { PDGFA } \\
\text { SPP1 } \\
\text { ANGPTL4 } \\
\text { IL1R2 } \\
\text { NR3C2 } \\
\text { PTGER3 } \\
\text { RORA } \\
\text { TUBB3 } \\
\text { ICOS }\end{array}$ & $\begin{array}{r}\text { P value } \\
<0.001 \\
<0.001 \\
<0.001 \\
<0.001 \\
0.047 \\
<0.001 \\
<0.001 \\
<0.001 \\
<0.001 \\
<0.001 \\
<0.001 \\
<0.001 \\
<0.001 \\
<0.001 \\
<0.001 \\
<0.001 \\
0.015 \\
<0.001 \\
<0.001 \\
<0.001 \\
<0.001 \\
0.020 \\
<0.001 \\
<0.001 \\
<0.001 \\
<0.001 \\
<0.001 \\
<0.001 \\
0.022 \\
<0.001 \\
<0.001 \\
<0.001 \\
0.041 \\
0.020 \\
<0.001 \\
<0.001 \\
<0.001\end{array}$ &  \\
\hline
\end{tabular}



Figure 3 Forest plot of the differentially-expressed IRGs related to prognosis. IRGs, immune-related genes.

build a five-IRG-based prognostic signature to predict the survival time of patients with IPF in the training cohort.

Figure $4 A-4 E$ shows the survival outcomes of IPF patients stratified by CXCL14, SLC40A1, RNASE3, CCR3, and $R O R A$. The survival curve revealed that IPF patients with higher expression levels of CXCL14, SLC4OA1, RNASE3, and CCR 3 had much worse survival outcomes. Patients with a relatively lower expression of RORA had markedly longer OS.

Detailed results of the multivariate Cox regression analysis, including coefficients, $\mathrm{P}$ values, hazard ratios, etc., are provided in Table S2. Accordingly, the patient's risk score representing the risk for OS was calculated as follows: risk score $=0.1970 \times$ expression value of CXCL14 +0.3280 $\times$ expression value of $S L C 40 A 1+0.5852 \times$ expression value of $R N A S E 3+0.2802 \times$ expression value of $C C R 3-0.6504$ $\times$ expression value of $R O R A$. According to the median risk score, IPF patients were divided into high- and low-risk groups. Individuals with risk scores beyond 0.711 were recognized as high-risk; otherwise, they were considered lowrisk (Figure 5 A, Table $\mathrm{S} 3$ ). There was a significant decrease in the OS of IPF patients as the risk score increased (Figure $5 B$ ). Figure $5 C$ displays the expression level of the five IRGs between the high- and low-risk groups. As shown in Figure 5C, CXCL14, SLC4OA1, RNASE3, and CCR3 were more highly expressed, while RORA expression exhibited relatively lower expression in the high-risk IPF patients than in the low-risk individuals. The survival curve constructed by the five-IRG-based prognostic signature in the training cohort showed that there was an extremely significant difference between the high- and low-risk groups (Figure 6A). A validation cohort was utilized to verify the five-IRG-based signature, and notable differential survival outcomes were observed between the high- and low-risk groups (Figure 6B). The area under curves (AUC) of the five-IRG-based prognostic signature for IPF in the training model was 0.858 (Figure $6 C$ ). The AUC of this predictive five-gene-based signature in the validation was 0.837 (Figure 6D), indicating that this predictive signature could be trusted.

\section{Correlation expression map of the five genes included in the predictive signature}

A correlation map of the five included prognostic IRGs expression levels is described in Figure 7. The strongest expression correlations were observed between RNASE3 

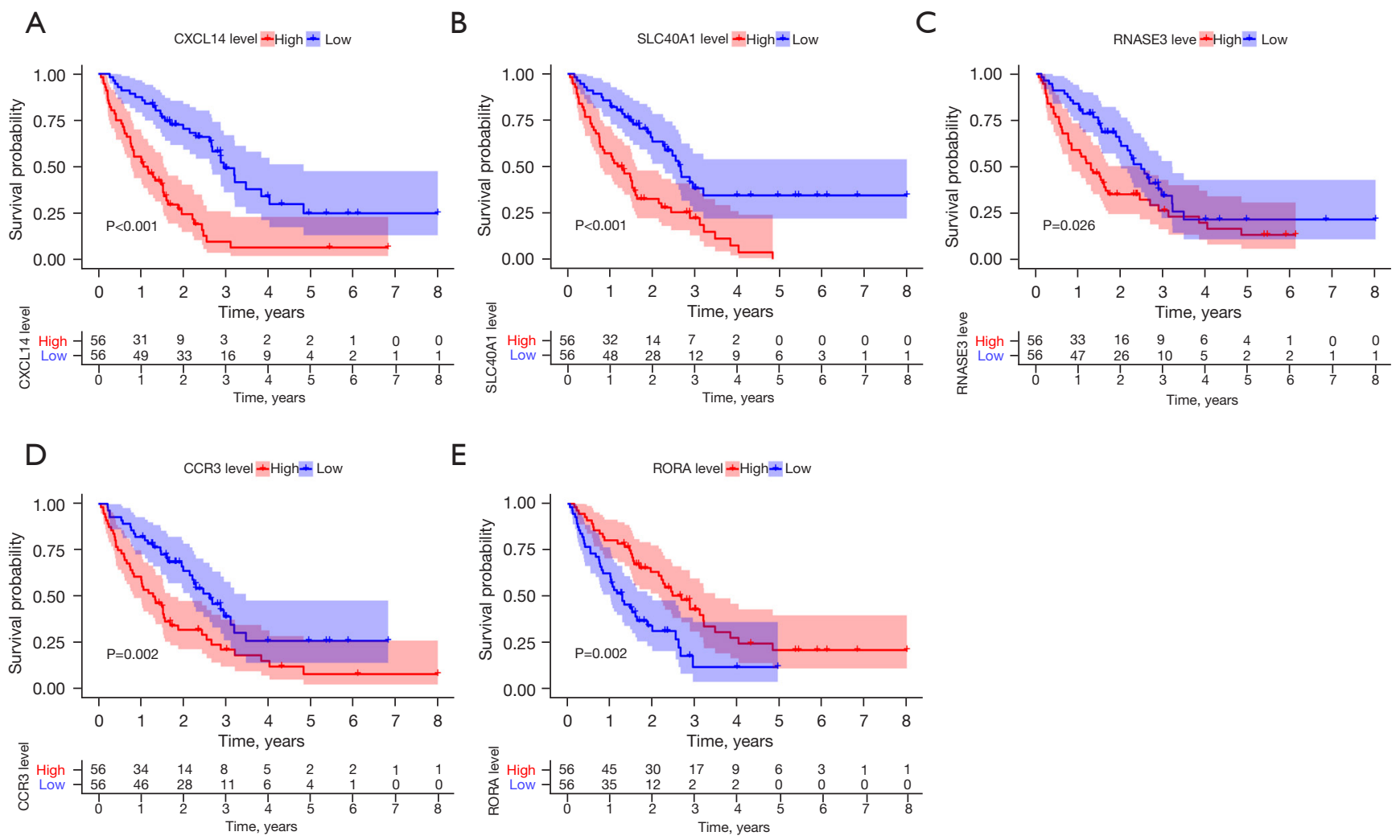

Figure 4 OS of patients with IPF stratified by the genes included in our novel signature, including (A) CXCL14, (B) SLC40A1, (C) RNASE3, (D) CCR3, and (E) RORA. OS, overall survival; IPF, idiopathic pulmonary fibrosis.

and SLC40A1 $(\mathrm{P}<0.01, \mathrm{r}=0.394)$, as well as between RORA and CXCL14 $(\mathrm{P}<0.01, \mathrm{r}=-0.355)$. Meanwhile, the expression level of $C C R 3$ was significantly positively correlated with the expression of CXCL14 ( $\mathrm{P}<0.01, \mathrm{r}=0.258)$. There was an intimate positive association between RNASE3 and CCR3 $(\mathrm{P}<0.01, \mathrm{r}=0.293)$.

\section{Discussion}

IPF is the most prevalent subtype of interstitial lung disease (ILD) worldwide (25). However, it has the poorest prognosis among the various ILD subtypes, with a median survival of 2-3 years after diagnosis $(3,4)$. Lung transplantation is the only intervention that has been shown to prolong survival for patients with IPF (26). Pirfenidone and nintedanib have emerged as effective therapies that can significantly slow the decline in forced vital capacity (FVC) and disease progression in IPF patients $(27,28)$. However, the prognosis of IPF remains unfavourable. The poor prognosis of IPF is partly due to a lack of effective prognostic biomarkers to guide treatment. Without the ability to forecast disease progression, it is difficult to determine which IPF patients are likely to benefit from new therapies or lung transplantation. Therefore, we constructed a molecular genomic signature to predict the prognosis of IPF patients using the GSE70866 gene expression dataset from the GEO database.

Previous studies have revealed that the immune system possesses an actual effect on the IPF process $(22,29,30)$. All stages of fibrogenesis are accompanied by innate and adaptive immune responses (22). More importantly, increasing evidence has appeared over the last few years establishing the meaningful role of IRGs in the pathogenesis and treatment of lung fibrosis $(23,24,31,32)$. It has been shown that regulating the expression of IRGs can ameliorate pulmonary fibrogenesis in bleomycin-induced (BLMinduced) mouse models $(31,32)$. Furthermore, data from clinical trials of newly developed drugs for the treatment of IPF have demonstrated the active role of IRG-targeting drugs in slowing disease progression. For instance, IRG- 
A

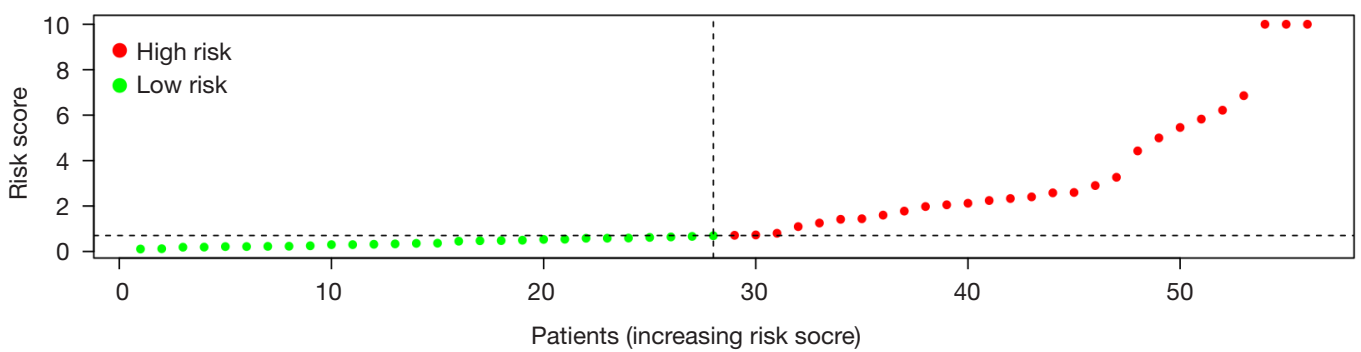

B



C

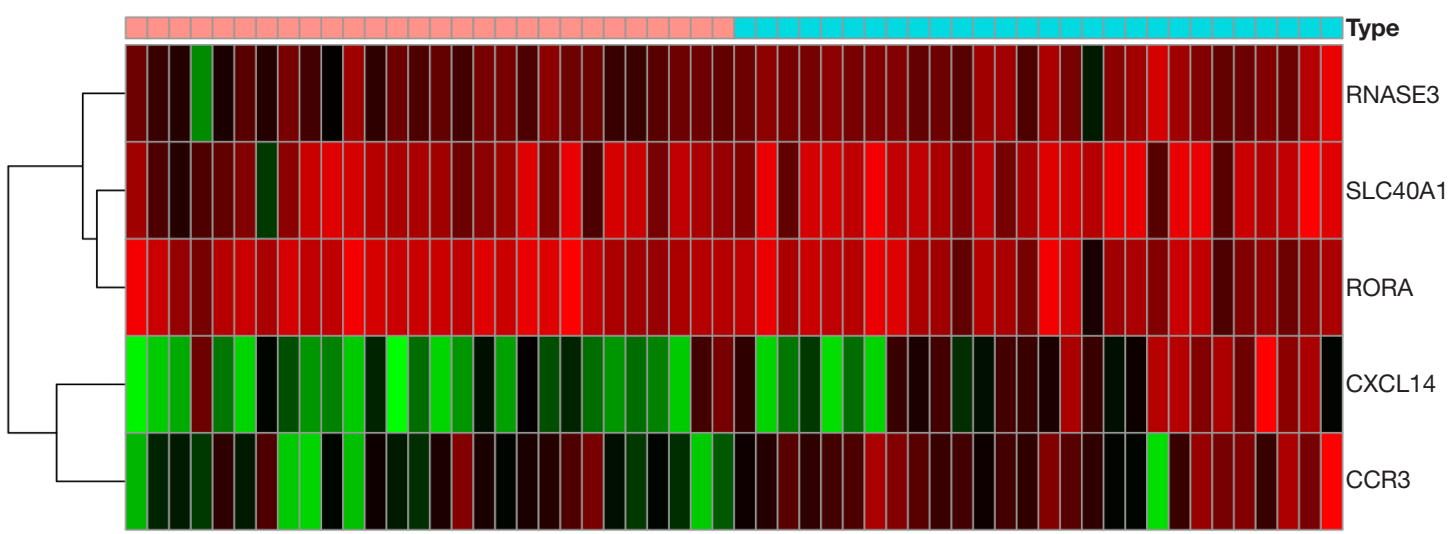

Figure 5 The risk score could effectively predict IPF patient prognosis. (A) Scatter plot of the risk score distribution of the samples. One point refers to a sample, red points are samples with higher risk scores, green points are samples with lower risk scores, and the intersecting point represents the median risk score. (B) Scatter plot of the survival outcome distribution of the samples. One point refers to a sample, red points represent live samples, green points represent dead samples with lower risk scores, and the intersecting point represents the median risk score. (C) Heatmap of signature-based genes (CXCL14, SLC40A1, RNASE3, CCR3, and RORA) between the high- and low-risk groups. IPF, idiopathic pulmonary fibrosis.

targeting drugs have been shown to play a positive role in reducing fibrogenesis (33). These previous studies highlight the importance of IRGs in the pathophysiological mechanism of IPF. In the present study, we were interested in the role of IRGs in the prognosis of IPF.

In total, 112 IPF patients and 20 healthy individuals were included in our study. The included IPF patients were predominantly older males (aged $>65$ years old). This demographic feature, as well as the fact that the prevalence of IPF is higher in men than in women, are consistent with previous studies $(1,3)$. In this comparative microarray profile of an IPF cohort versus a healthy individual cohort, a total of 379 DEGs were identified. The genes involved in encoding extracellular matrix (ECM) components, tissue architecture remodeling, and ECM accumulation (SPP1, MMP7, MMP10, CCL2, and ITGB3) were observed to be significantly upregulated (34-37). Of the 379 DEGs, 52 were filtered as IRGs based on the ImmPort database. 
A

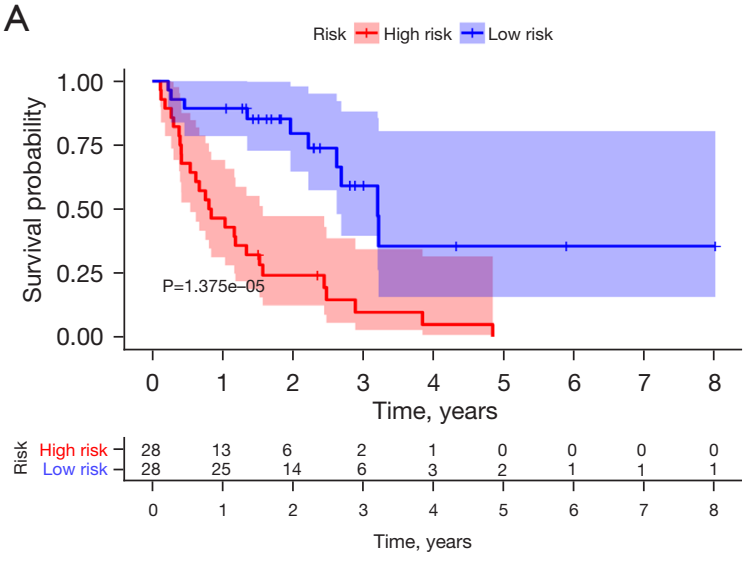

C

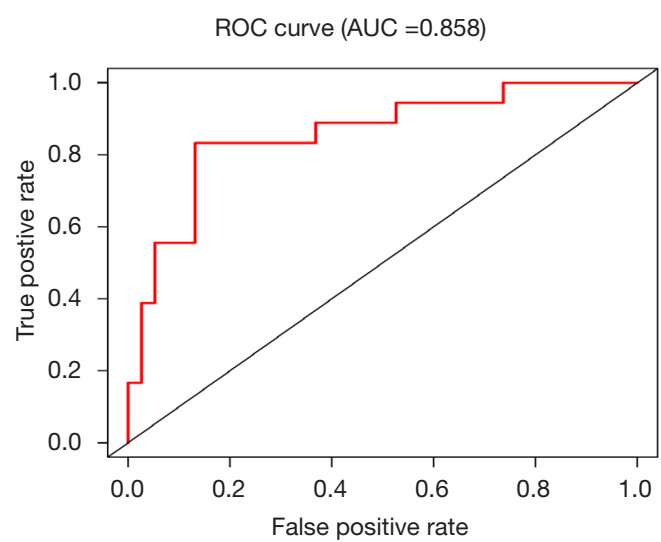

B

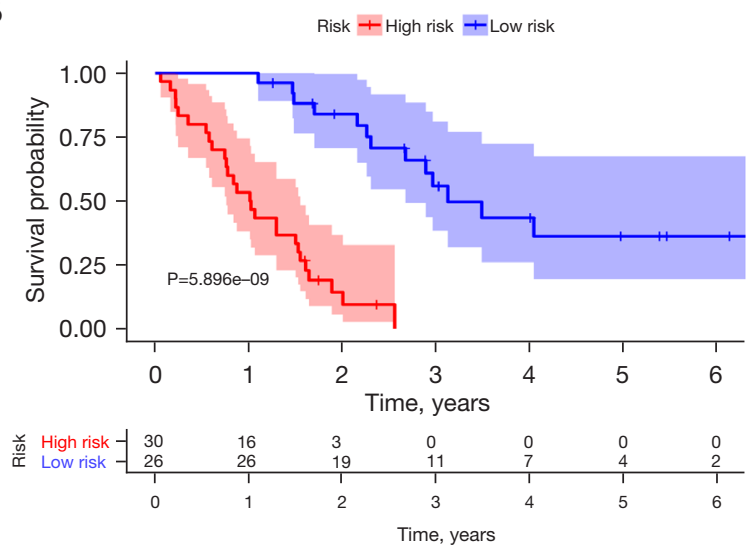

$\mathrm{D}$

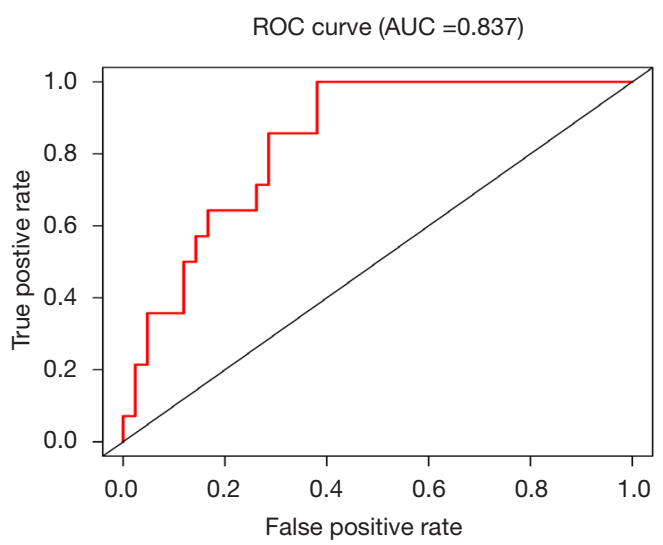

Figure 6 Signature of predicting survival probability for IPF patients. (A) Survival curve of the risk score distribution of the training cohort, which also shows the 1-, 2-, 3-, 4-, 5-, and 6-year survival rates of IPF patients. (B) Survival curve of the risk score distribution of the validation cohort, which also shows the 1-, 2-, 3-, 4-, 5-, and 6-year survival rates of IPF patients. (C) ROC curve of the signature in the training cohort. (D) ROC curve of the signature in the validation cohort. IPF, idiopathic pulmonary fibrosis; ROC, receiver operating characteristic.

Next, 37 of these 52 differentially-expressed IRGs were recognized as significant prognostic biomarkers for patients with IPF. More than $70 \%$ of the differentially-expressed IRGs had notable associations with survival. Our results further suggested that there was a close association between IRGs and the progression of IPF, which was consistent with previous studies. Based on these findings, a five IRG-based prognostic signature (CXCL14, SLC40A1, RNASE3, CCR3, and $R O R A$ ), was built in the training cohort in this study. This signature presented an excellent predictive prognostic effect, with an AUC value of 0.858 . In addition, the risk score was significantly different between the high- and lowrisk groups. Meanwhile, the risk score was significantly correlated with the OS of IPF patients. CXCL14, SLC40A1,
CXCL14, and CCR3 were differentially-upregulated genes between IPF patients and healthy individuals. The expression levels of these four genes in the high-risk IPF group were significantly higher than those in the low-risk group. RORA was detected at a lower expression level in the healthy individuals group compared to the IPF group. Consistently, the expression level of RORA was lower in the high-risk IPF group than in the low-risk group.

Fibroblast foci represent the main pathogenic lesions of IPF, including abnormally activated fibroblasts and myofibroblasts. Myofibroblasts are the main effector cells of IPF. They can secrete a large amount of ECM protein and promote the abnormal hardening of ECM, which leads to the remodeling of lung structure and the 


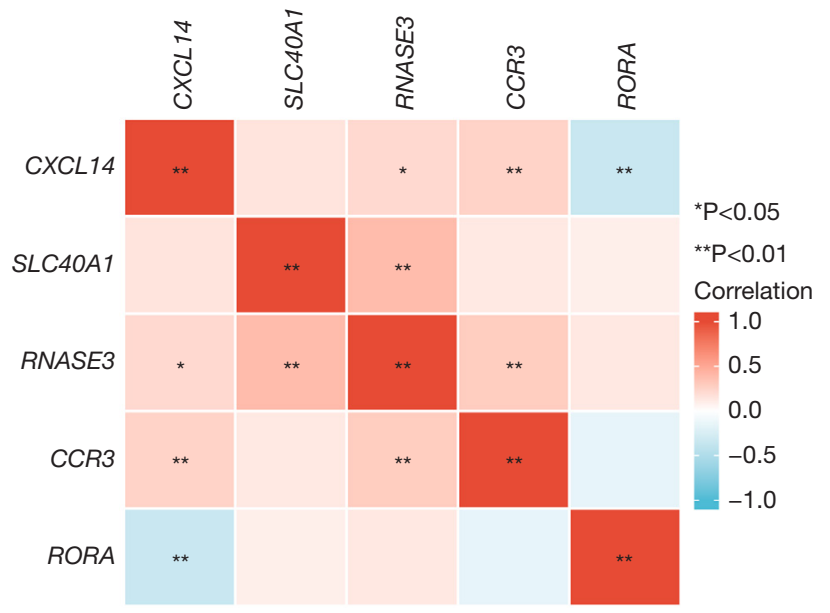

Figure 7 Gene co-expression network of 5 genes: CXCL14, SLC4OA1, RNASE3, CCR3, and RORA.

gradual loss of lung function (38-40). Previous studies have confirmed that knockdown of CXCL14 could inhibit lung fibrogenesis by suppressing lung fibroblasts proliferation and downregulating $M M P 2 / 9$ (31). Zagai et al. found eosinophil cationic protein (ECP, also known as $R N A S E 3)$ could stimulate human lung fibroblasts to secrete extracellular matrix, thereby leads to airway fibrosis (41). The concentration of RNASE3 in bronchoalveolar lavage fluid (BALF) is markedly increased in IPF patients compared with healthy individuals and is highly correlated with acute exacerbation during the preceding 3- to 6-month period $(42,43) . C C R 3$ can increase the activation, migration and proliferation ability of lung fibroblasts, and the ability of myofibroblasts to secrete ECM protein $(44,45)$. In addition, CCR3 is notably expressed in the lungs of BLMinduced mice and is expressed not only by eosinophils but also by neutrophils (44). CCR3 plays a key role in the recruitment of granulocytes and is an important suppressor of fibrogenesis in BLM-treated lungs (44). These studies on the pathophysiological mechanisms between IPF and CXCL14, RNASE3, and CCR3 increase the credibility of the signature constructed in our study. Our research also showed that there is a meaningful correlation between the expression of RNASE3 and CCR3. Meanwhile, a significant expression correlation between CXCL14 and CCR 3 was also observed in this study. For the SLC4OA1 and RORA, no relevant studies have been conducted to determine the association with lung fibrosis. We first reported that there may be some potential associations between the pathological mechanism of IPF and SLC4OA1 along with RORA. The specific pathophysiological mechanism is worthy of further study.

Finally, we evaluated the performance of the genomic signature in the validation cohort. The signature showed an equally excellent ability to distinguish between high- and low-risk patient groups. The AUC value of the Receiver Operating Characteristic Curve (ROC) curve was 0.837 , demonstrating the potential applicability of our findings for real-world use.

While the genomic model developed in this study was successfully validated, there were still some potential limitations that should be noted. Firstly, this research was based on the gene expression profiles from the GEO database. Due to the difficult of recruitment of a large number of IPF patients, no validation of the 5 genes in real world data in this paper. Also, the IPF patients included in this study were all from Germany. Thus, our results might only represent patients in Germany and might not applicable to all IPF patients worldwide. Finally, due to limited data on treatment, our study did not subgroup IPF patients according to the different treatment choices. Consequently, the reliability and accuracy of our results might be affected and needs to be re-evaluated by future studies.

\section{Conclusions}

In conclusion, our study identified a novel five-IRG-based signature that is a reproducible predictor of outcome in IPF patients. This novel signature benefits the personalized management of patients with IPF. Furthermore, this finding provides new insights into the relationship between the immune system and IPF, offering incremental clinical value for IPF prognosis and therapy.

\section{Acknowledgments}

We acknowledge GEO database for providing their platforms and contributors for uploading their meaningful datasets.

Funding: This study was supported by grants from the Henan Provincial Key Laboratory for Interstitial Lung Disease and Lung Transplantation.

\section{Footnote}

Reporting Checklist: The authors have completed the STARD reporting checklist. Available at https://dx.doi. 
org/10.21037/atm-21-4545

Conflicts of Interest: All authors have completed the ICMJE uniform disclosure form (available at https://dx.doi. org/10.21037/atm-21-4545). The authors have no conflicts of interest to declare.

Ethical Statement: The authors are accountable for all aspects of the work in ensuring that questions related to the accuracy or integrity of any part of the work are appropriately investigated and resolved. The study was conducted in accordance with the Declaration of Helsinki (as revised in 2013). The data used in this study was derived from a public database, and thus, no ethical approval was needed.

Open Access Statement: This is an Open Access article distributed in accordance with the Creative Commons Attribution-NonCommercial-NoDerivs 4.0 International License (CC BY-NC-ND 4.0), which permits the noncommercial replication and distribution of the article with the strict proviso that no changes or edits are made and the original work is properly cited (including links to both the formal publication through the relevant DOI and the license). See: https://creativecommons.org/licenses/by-nc-nd/4.0/.

\section{References}

1. Raghu G, Remy-Jardin M, Myers JL, et al. Diagnosis of Idiopathic Pulmonary Fibrosis. An Official ATS/ERS/JRS/ ALAT Clinical Practice Guideline. Am J Respir Crit Care Med 2018;198:e44-68.

2. Selman M, King TE, Pardo A, et al. Idiopathic pulmonary fibrosis: prevailing and evolving hypotheses about its pathogenesis and implications for therapy. Ann Intern Med 2001;134:136-51.

3. Raghu G, Collard HR, Egan JJ, et al. An official ATS/ ERS/JRS/ALAT statement: idiopathic pulmonary fibrosis: evidence-based guidelines for diagnosis and management. Am J Respir Crit Care Med 2011;183:788-824.

4. Mura M, Porretta MA, Bargagli E, et al. Predicting survival in newly diagnosed idiopathic pulmonary fibrosis: a 3-year prospective study. Eur Respir J 2012;40:101-9.

5. Vancheri C, du Bois RM. A progression-free end-point for idiopathic pulmonary fibrosis trials: lessons from cancer. Eur Respir J 2013;41:262-9.

6. Kim HJ, Perlman D, Tomic R. Natural history of idiopathic pulmonary fibrosis. Respir Med 2015;109:661-70.
7. Kim DS, Collard HR, King TE Jr. Classification and natural history of the idiopathic interstitial pneumonias. Proc Am Thorac Soc 2006;3:285-92.

8. Ley B, Collard HR, King TE Jr. Clinical course and prediction of survival in idiopathic pulmonary fibrosis. Am J Respir Crit Care Med 2011;183:431-40.

9. Selman M, López-Otín C, Pardo A. Age-driven developmental drift in the pathogenesis of idiopathic pulmonary fibrosis. Eur Respir J 2016;48:538-52.

10. Bueno M, Lai YC, Romero Y, et al. PINK1 deficiency impairs mitochondrial homeostasis and promotes lung fibrosis. J Clin Invest 2015;125:521-38.

11. Prasse A, Binder H, Schupp JC, et al. BAL Cell Gene Expression Is Indicative of Outcome and Airway Basal Cell Involvement in Idiopathic Pulmonary Fibrosis. Am J Respir Crit Care Med 2019;199:622-30.

12. Caporarello N, Meridew JA, Jones DL, et al. PGC1 $\alpha$ repression in IPF fibroblasts drives a pathologic metabolic, secretory and fibrogenic state. Thorax 2019;74:749-60.

13. McDonough JE, Kaminski N, Thienpont B, et al. Gene correlation network analysis to identify regulatory factors in idiopathic pulmonary fibrosis. Thorax 2019;74:132-40.

14. DePianto DJ, Chandriani S, Abbas AR, et al. Heterogeneous gene expression signatures correspond to distinct lung pathologies and biomarkers of disease severity in idiopathic pulmonary fibrosis. Thorax 2015;70:48-56.

15. Boon K, Bailey NW, Yang J, et al. Molecular phenotypes distinguish patients with relatively stable from progressive idiopathic pulmonary fibrosis (IPF). PLoS One 2009;4:e5134.

16. Korfei M, von der Beck D, Henneke I, et al. Comparative proteome analysis of lung tissue from patients with idiopathic pulmonary fibrosis (IPF), non-specific interstitial pneumonia (NSIP) and organ donors. J Proteomics 2013;85:109-28.

17. Xu Z, Mo L, Feng X, et al. Using bioinformatics approach identifies key genes and pathways in idiopathic pulmonary fibrosis. Medicine (Baltimore) 2020;99:e22099.

18. Min F, Gao F, Liu Z. Screening and further analyzing differentially expressed genes in acute idiopathic pulmonary fibrosis with DNA microarray. Eur Rev Med Pharmacol Sci 2013;17:2784-90.

19. Li FJ, Surolia R, Li H, et al. Autoimmunity to Vimentin Is Associated with Outcomes of Patients with Idiopathic Pulmonary Fibrosis. J Immunol 2017;199:1596-605.

20. Huang Y, Ma SF, Vij R, et al. A functional genomic model for predicting prognosis in idiopathic pulmonary fibrosis. BMC Pulm Med 2015;15:147.

21. Meyer KC, Raghu G, Baughman RP, et al. An official 
American Thoracic Society clinical practice guideline: the clinical utility of bronchoalveolar lavage cellular analysis in interstitial lung disease. Am J Respir Crit Care Med 2012;185:1004-14.

22. Heukels P, Moor CC, von der Thüsen JH, et al. Inflammation and immunity in IPF pathogenesis and treatment. Respir Med 2019;147:79-91.

23. Cecchini MJ, Hosein K, Howlett CJ, et al. Comprehensive gene expression profiling identifies distinct and overlapping transcriptional profiles in non-specific interstitial pneumonia and idiopathic pulmonary fibrosis. Respir Res 2018;19:153.

24. Walsh SM, Worrell JC, Fabre A, et al. Novel differences in gene expression and functional capabilities of myofibroblast populations in idiopathic pulmonary fibrosis. Am J Physiol Lung Cell Mol Physiol 2018;315:L697-710.

25. Lederer DJ, Martinez FJ. Idiopathic Pulmonary Fibrosis. N Engl J Med 2018;378:1811-23.

26. George PM, Patterson CM, Reed AK, et al. Lung transplantation for idiopathic pulmonary fibrosis. Lancet Respir Med 2019;7:271-82.

27. Richeldi L, Costabel U, Selman M, et al. Efficacy of a tyrosine kinase inhibitor in idiopathic pulmonary fibrosis. N Engl J Med 2011;365:1079-87.

28. Taniguchi H, Ebina M, Kondoh Y, et al. Pirfenidone in idiopathic pulmonary fibrosis. Eur Respir J 2010;35:821-9.

29. Drakopanagiotakis F, Wujak L, Wygrecka M, et al. Biomarkers in idiopathic pulmonary fibrosis. Matrix Biol 2018;68-69:404-21.

30. O'Dwyer DN, Ashley SL, Moore BB. Influences of innate immunity, autophagy, and fibroblast activation in the pathogenesis of lung fibrosis. Am J Physiol Lung Cell Mol Physiol 2016;311:L590-601.

31. Li L, Li Q, Wei L, et al. Chemokine (C-X-C motif) ligand 14 contributes to lipopolysaccharide-induced fibrogenesis in mouse L929 fibroblasts via modulating PPM1A. J Cell Biochem 2019;120:13372-81.

32. Mehrad B, Burdick MD, Strieter RM. Fibrocyte CXCR4 regulation as a therapeutic target in pulmonary fibrosis. Int J Biochem Cell Biol 2009;41:1708-18.

33. Lukey PT, Harrison SA, Yang S, et al. A randomised, placebo-controlled study of omipalisib (PI3K/ mTOR) in idiopathic pulmonary fibrosis. Eur Respir J 2019;53:1801992.

34. Insua-Rodríguez J, Pein M, Hongu T, et al. Stress signaling in breast cancer cells induces matrix components that promote chemoresistant metastasis. EMBO Mol Med 2018;10:e9003.

35. Rohani MG, Parks WC. Matrix remodeling by MMPs during wound repair. Matrix Biol 2015;44-46:113-21.

36. Kuo CS, Pavlidis S, Zhu J, et al. Contribution of airway eosinophils in airway wall remodeling in asthma: Role of MMP-10 and MET. Allergy 2019;74:1102-12.

37. Liu A, Liu Y, Li B, et al. Role of miR-223-3p in pulmonary arterial hypertension via targeting ITGB3 in the ECM pathway. Cell Prolif 2019;52:e12550.

38. Guillotin D, Taylor AR, Platé M, et al. Transcriptome analysis of IPF fibroblastic foci identifies key pathways involved in fibrogenesis. Thorax 2021;76:73-82.

39. Richeldi L, Collard HR, Jones MG. Idiopathic pulmonary fibrosis. Lancet 2017;389:1941-52.

40. Martinez FJ, Collard HR, Pardo A, et al. Idiopathic pulmonary fibrosis. Nat Rev Dis Primers 2017;3:17074.

41. Zagai U, Dadfar E, Lundahl J, et al. Eosinophil cationic protein stimulates TGF-beta 1 release by human lung fibroblasts in vitro. Inflammation 2007;30:153-60.

42. Fujimoto K, Kubo K, Yamaguchi S, et al. Eosinophil activation in patients with pulmonary fibrosis. Chest 1995;108:48-54.

43. Birring SS, Parker D, McKenna S, et al. Sputum eosinophilia in idiopathic pulmonary fibrosis. Inflamm Res 2005;54:51-6.

44. Huaux F, Gharaee-Kermani M, Liu T, et al. Role of Eotaxin-1 (CCL11) and CC chemokine receptor 3 (CCR3) in bleomycin-induced lung injury and fibrosis. Am J Pathol 2005;167:1485-96.

45. Puxeddu I, Bader R, Piliponsky AM, et al. The CC chemokine eotaxin/CCL11 has a selective profibrogenic effect on human lung fibroblasts. J Allergy Clin Immunol 2006;117:103-10.

(English Language Editor: B. Draper)

Cite this article as: Qiu L, Gong G, Wu W, Li N, Li Z, Chen S, Li P, Chen T, Zhao H, Hu C, Fang Z, Wang Y, Liu H, Cui P, Zhang G. A novel prognostic signature for idiopathic pulmonary fibrosis based on five-immune-related genes. Ann Transl Med 2021;9(20):1570. doi: 10.21037/atm-214545 


\section{Supplementary}

Table S1 The information of $\log \mathrm{FC}$ for differential expression genes between IPF patients and the control group

\begin{tabular}{|c|c|}
\hline id & $\log F C$ \\
\hline SPP1 & 3.856003 \\
\hline PPBP & 3.767079 \\
\hline MMP7 & 3.094781 \\
\hline SFTPB & 3.001383 \\
\hline ITGB3 & 2.777328 \\
\hline CYP1B1 & 2.489336 \\
\hline TUBB3 & 2.463291 \\
\hline LRRC2 & 2.409921 \\
\hline CYTL1 & 2.379117 \\
\hline VSNL1 & 2.262473 \\
\hline HTRA1 & 2.239659 \\
\hline OLIG1 & 2.238058 \\
\hline TIMP3 & 2.238012 \\
\hline FFAR3 & 2.235872 \\
\hline CCL2 & 2.19958 \\
\hline MMP10 & 2.157871 \\
\hline MERTK & 2.138287 \\
\hline C14orf34 & 2.129425 \\
\hline S100A12 & 2.119374 \\
\hline BICC1 & 2.10942 \\
\hline GPR179 & 2.106344 \\
\hline PLA2G7 & 2.106114 \\
\hline FABP3 & 2.102401 \\
\hline DEFA3 & 2.095526 \\
\hline SPINK1 & 2.068789 \\
\hline TPSAB1 & 2.048006 \\
\hline FNDC5 & 2.003073 \\
\hline IL1R2 & 2.000731 \\
\hline AQP4 & 1.980216 \\
\hline SOD3 & 1.972751 \\
\hline STAB1 & 1.966629 \\
\hline SFTPC & 1.939547 \\
\hline TPSD1 & 1.936252 \\
\hline CCL7 & 1.932093 \\
\hline
\end{tabular}

Table S1 (continued)
Table S1 (continued)

\begin{tabular}{lc}
\hline id & logFC \\
\hline VSTM1 & 1.917281 \\
TPST1 & 1.913652 \\
PROM2 & 1.905313 \\
CST6 & 1.888228 \\
SDS & 1.879609 \\
WNT2B & 1.874928 \\
GPR182 & 1.869439 \\
HBD & 1.86808 \\
HS3ST2 & 1.837265 \\
ANGPTL4 & 1.76816 \\
LOC729040 & 1.764131 \\
TM4SF1 & 1.762285 \\
GFRA2 & 1.757664 \\
MATK & 1.744541 \\
EMP1 & 1.734211 \\
AANAT & 1.710304 \\
RNASE1 & 1.678622 \\
DACH1 & 1.675864 \\
COL22A1 & 1.675523 \\
NRAP & 1.67189 \\
PID1 & 1.667208 \\
CCR3 & 1.661399 \\
KIAA0125 & 1.653681 \\
F13A1 & 1.652466 \\
CPA3 & 1.649287 \\
CD86 & 1.637381 \\
RPA4 & 1.625746 \\
ARAP3 & 1.624116 \\
\hline NT5DC28A3 & 1.605387 \\
RNASE2 & 1.602076 \\
EHTH & 1.596596 \\
\hline
\end{tabular}

Table S1 (continued) 
Table S1 (continued)

\begin{tabular}{|c|c|}
\hline id & $\log F C$ \\
\hline MGC24103 & 1.580791 \\
\hline CXCL14 & 1.5796 \\
\hline $\mathrm{CH} 25 \mathrm{H}$ & 1.575003 \\
\hline RGL1 & 1.550247 \\
\hline MRVI1 & 1.542015 \\
\hline RAB3IL1 & 1.53331 \\
\hline SEPP1 & 1.528336 \\
\hline OR13H1 & 1.516111 \\
\hline KRT79 & 1.498058 \\
\hline MALL & 1.495627 \\
\hline IBSP & 1.492416 \\
\hline ADM & 1.489362 \\
\hline $\mathrm{PI} 3$ & 1.479423 \\
\hline STEAP4 & 1.46926 \\
\hline CLC & 1.467909 \\
\hline CCL13 & 1.458057 \\
\hline CDA & 1.445885 \\
\hline CCL26 & 1.442975 \\
\hline ARNT2 & 1.43881 \\
\hline DIRAS1 & 1.426543 \\
\hline HDC & 1.416884 \\
\hline CLGN & 1.408777 \\
\hline HS3ST1 & 1.408676 \\
\hline PRSS8 & 1.406913 \\
\hline HIST2H3A & 1.403166 \\
\hline GPT & 1.382998 \\
\hline C10orf116 & 1.376584 \\
\hline IL8 & 1.373865 \\
\hline $\mathrm{CNIH} 3$ & 1.373499 \\
\hline CMKLR1 & 1.37083 \\
\hline ACOX2 & 1.367016 \\
\hline SH3RF1 & 1.365489 \\
\hline RNASE3 & 1.362714 \\
\hline MRC2 & 1.355777 \\
\hline
\end{tabular}

Table S1 (continued)
Table S1 (continued)

\begin{tabular}{|c|c|}
\hline id & $\log \mathrm{FC}$ \\
\hline LGMN & 1.352665 \\
\hline CD36 & 1.350796 \\
\hline MPO & 1.337994 \\
\hline CYR61 & 1.330317 \\
\hline ASPHD1 & 1.325651 \\
\hline KRT14 & 1.291002 \\
\hline TM4SF19 & 1.288525 \\
\hline RGS2 & 1.27315 \\
\hline CACNA1G & 1.272916 \\
\hline OR8G5 & 1.27172 \\
\hline FCN1 & 1.267912 \\
\hline IER3 & 1.264788 \\
\hline KIT & 1.254733 \\
\hline TDRD10 & 1.254632 \\
\hline PRKAR1B & 1.240034 \\
\hline VCAN & 1.234562 \\
\hline MMP9 & 1.227406 \\
\hline PCSK9 & 1.212719 \\
\hline MS4A2 & 1.211344 \\
\hline AREG & 1.20871 \\
\hline SFTPD & 1.206889 \\
\hline FAM20A & 1.20638 \\
\hline ECM1 & 1.206065 \\
\hline CEACAM7 & 1.202738 \\
\hline SNAI1 & 1.199136 \\
\hline HRK & 1.196395 \\
\hline KCNG1 & 1.194507 \\
\hline CLDN18 & 1.193072 \\
\hline CXCL5 & 1.192979 \\
\hline SLC40A1 & 1.19176 \\
\hline DIRC3 & 1.190271 \\
\hline ATP9A & 1.182874 \\
\hline FBXO15 & 1.181945 \\
\hline P2RY2 & 1.180127 \\
\hline
\end{tabular}

Table S1 (continued) 
Table S1 (continued)

\begin{tabular}{|c|c|}
\hline id & $\log F C$ \\
\hline FGFR1 & 1.177801 \\
\hline PGA3 & 1.176969 \\
\hline LOC100132368 & 1.170005 \\
\hline SFN & 1.16869 \\
\hline MUC21 & 1.166485 \\
\hline HOMER3 & 1.164147 \\
\hline S1PR3 & 1.162823 \\
\hline HAMP & 1.160114 \\
\hline SPTLC3 & 1.159878 \\
\hline ABLIM3 & 1.156865 \\
\hline ENHO & 1.155081 \\
\hline AQP2 & 1.154688 \\
\hline SLC16A10 & 1.152375 \\
\hline SEC14L2 & 1.148491 \\
\hline SLC24A3 & 1.145543 \\
\hline LTC4S & 1.145343 \\
\hline TAAR2 & 1.143163 \\
\hline LRG1 & 1.139364 \\
\hline C6orf108 & 1.139162 \\
\hline HIST1H3B & 1.137506 \\
\hline GAS6 & 1.134418 \\
\hline SULT1C2 & 1.131406 \\
\hline DYSF & 1.126893 \\
\hline C1orf111 & 1.126254 \\
\hline LOC283050 & 1.123883 \\
\hline HES4 & 1.119226 \\
\hline KRT17 & 1.1121 \\
\hline CALB2 & 1.110349 \\
\hline MUC1 & 1.110008 \\
\hline NRGN & 1.106171 \\
\hline EPO & 1.103573 \\
\hline PAX6 & 1.100351 \\
\hline FAM198B & 1.098049 \\
\hline NIPAL4 & 1.092226 \\
\hline
\end{tabular}

Table S1 (continued)
Table S1 (continued)

\begin{tabular}{|c|c|}
\hline id & $\log \mathrm{FC}$ \\
\hline GAL3ST4 & 1.086711 \\
\hline NOV & 1.086149 \\
\hline CYBRD1 & 1.086021 \\
\hline SNCA & 1.085304 \\
\hline SPTB & 1.08173 \\
\hline FCGR2B & 1.080386 \\
\hline CLEC5A & 1.075267 \\
\hline CXCL1 & 1.074218 \\
\hline QPCT & 1.072253 \\
\hline C14orf162 & 1.070768 \\
\hline OR52E8 & 1.066605 \\
\hline FAM124B & 1.06621 \\
\hline UCK2 & 1.064365 \\
\hline MGC14436 & 1.063247 \\
\hline SLC16A8 & 1.05739 \\
\hline FCER2 & 1.056397 \\
\hline PPP1R14C & 1.053759 \\
\hline IL1RN & 1.051554 \\
\hline CLEC11A & 1.046712 \\
\hline PMP22 & 1.041398 \\
\hline SFRP1 & 1.03858 \\
\hline SFTA2 & 1.034844 \\
\hline MYL9 & 1.034835 \\
\hline NPAS2 & 1.030934 \\
\hline CD24 & 1.030668 \\
\hline LEPREL1 & 1.030111 \\
\hline LOC284263 & 1.02944 \\
\hline SFTPA2 & 1.029373 \\
\hline MGP & 1.024552 \\
\hline CEBPE & 1.023772 \\
\hline MYO7A & 1.022615 \\
\hline FAM20C & 1.020749 \\
\hline KRTAP4-11 & 1.020715 \\
\hline LOC100130480 & 1.017798 \\
\hline
\end{tabular}

Table S1 (continued) 
Table S1 (continued)

\begin{tabular}{ll}
\hline id & logFC \\
\hline PDGFA & 1.012284 \\
SEMA3B & 1.00929 \\
KIF4A & 1.005653 \\
SLC47A1 & -1.00141 \\
ERN2 & -1.00479 \\
MPP7 & -1.00695 \\
HOXC4 & -1.00729 \\
GATA3 & -1.00734 \\
MYB & -1.00767 \\
RANBP3L & -1.00783 \\
RNF183 & -1.01242 \\
C110r880 & -1.01247 \\
CD6 & -1.01563 \\
JAG2 & -1.01796 \\
AQP7P3 & -1.02239 \\
LOC283392 & -1.02313 \\
LOC100270804 & -1.02639 \\
RORA & -1.02654 \\
SNTN & -1.02656 \\
HRASLS & -1.0302 \\
ITK & -1.03125 \\
SNAI2 & -1.03637 \\
SLC7A2 & -1.03645 \\
C8A & -1.03662 \\
CSPG4 & -1.03949 \\
LOC650293 & -1.04049 \\
TFRC & -1.04068 \\
RIC3 & -1.04191 \\
FNF404 & -1.04298 \\
NR3C2 & -1.04313 \\
THA2 & -1.04324 \\
\hline
\end{tabular}

Table S1 (continued)
Table S1 (continued)

\begin{tabular}{|c|c|}
\hline id & $\log \mathrm{FC}$ \\
\hline ZNF589 & -1.05218 \\
\hline SNORA12 & -1.05973 \\
\hline PM20D1 & -1.06203 \\
\hline TCF7 & -1.06253 \\
\hline EPB41L4A & -1.06331 \\
\hline TNNT1 & -1.06454 \\
\hline ZNF610 & -1.06512 \\
\hline SCN8A & -1.06542 \\
\hline ARMC3 & -1.06592 \\
\hline LOC256880 & -1.06671 \\
\hline D4S234E & -1.06734 \\
\hline LARP6 & -1.06889 \\
\hline IFT81 & -1.06903 \\
\hline SERPINI2 & -1.07138 \\
\hline LOC400655 & -1.07223 \\
\hline GPR85 & -1.07447 \\
\hline DLEC1 & -1.07639 \\
\hline ITGB8 & -1.0784 \\
\hline MYO7B & -1.08026 \\
\hline CDC42EP3 & -1.08253 \\
\hline LOC728218 & -1.08572 \\
\hline ABHD1 & -1.08959 \\
\hline MAL & -1.09019 \\
\hline MAGI3 & -1.09278 \\
\hline COL9A2 & -1.09321 \\
\hline KPNA5 & -1.10405 \\
\hline GRIN3B & -1.10515 \\
\hline DSP & -1.11168 \\
\hline KLK11 & -1.11497 \\
\hline LOC729867 & -1.11537 \\
\hline C7orf58 & -1.11548 \\
\hline TMEM130 & -1.11625 \\
\hline EPM2AIP1 & -1.11628 \\
\hline NDN & -1.12473 \\
\hline
\end{tabular}

Table S1 (continued) 
Table S1 (continued)

\begin{tabular}{|c|c|}
\hline id & $\log \mathrm{FC}$ \\
\hline ODZ4 & -1.1275 \\
\hline TPBG & -1.1293 \\
\hline CAPS2 & -1.12947 \\
\hline OR2A7 & -1.13147 \\
\hline RAP1GAP2 & -1.13156 \\
\hline FLJ46875 & -1.13275 \\
\hline ENPP5 & -1.13735 \\
\hline FAM3B & -1.13918 \\
\hline ICOS & -1.13931 \\
\hline C20orf46 & -1.14512 \\
\hline RAB39B & -1.15124 \\
\hline DNAH5 & -1.15318 \\
\hline FBXL16 & -1.15713 \\
\hline SLC4A8 & -1.1666 \\
\hline CAPN11 & -1.16854 \\
\hline ANK3 & -1.16942 \\
\hline SERPINB4 & -1.17668 \\
\hline GPRASP1 & -1.18083 \\
\hline LOC100131289 & -1.19614 \\
\hline NHS & -1.19903 \\
\hline MAP9 & -1.20022 \\
\hline CES1 & -1.20284 \\
\hline ZBP1 & -1.20287 \\
\hline ACSS3 & -1.20422 \\
\hline HLF & -1.2142 \\
\hline ZNF251 & -1.21519 \\
\hline AKR1E2 & -1.21823 \\
\hline FAM70A & -1.21997 \\
\hline CHRM2 & -1.22546 \\
\hline PDCD1LG2 & -1.23139 \\
\hline NBEA & -1.2346 \\
\hline TRIB2 & -1.23661 \\
\hline LOC400891 & -1.23772 \\
\hline SEC16B & -1.24531 \\
\hline
\end{tabular}

Table S1 (continued)
Table S1 (continued)

\begin{tabular}{|c|c|}
\hline id & $\log F C$ \\
\hline IQCA1 & -1.24651 \\
\hline ZMAT3 & -1.252 \\
\hline ZFP14 & -1.25298 \\
\hline MFAP3L & -1.25472 \\
\hline SYNE2 & -1.2653 \\
\hline KLF12 & -1.26866 \\
\hline KIAA0408 & -1.27245 \\
\hline FAM47E & -1.27394 \\
\hline LPAR3 & -1.27435 \\
\hline MYO1A & -1.27997 \\
\hline C17orf69 & -1.28199 \\
\hline EPB41L5 & -1.28237 \\
\hline TJP1 & -1.29275 \\
\hline ODF3L1 & -1.29365 \\
\hline RFPL4A & -1.30527 \\
\hline GDA & -1.31169 \\
\hline C9orf30-TMEFF1 & -1.31377 \\
\hline C9orf171 & -1.32157 \\
\hline SLITRK4 & -1.3288 \\
\hline TC2N & -1.33076 \\
\hline ACSM1 & -1.33541 \\
\hline PLEKHA6 & -1.33918 \\
\hline CPLX3 & -1.35549 \\
\hline KLRB1 & -1.35674 \\
\hline BEX5 & -1.35708 \\
\hline ZNF540 & -1.37252 \\
\hline IFNG & -1.37571 \\
\hline TRAT1 & -1.38275 \\
\hline XCL1 & -1.40778 \\
\hline DLX3 & -1.41913 \\
\hline TNFRSF25 & -1.42826 \\
\hline PIGR & -1.43529 \\
\hline LAMB1 & -1.43589 \\
\hline SAMD12 & -1.45227 \\
\hline
\end{tabular}

Table S1 (continued) 
Table S1 (continued)

\begin{tabular}{|c|c|}
\hline id & $\log F C$ \\
\hline CXCL9 & -1.46689 \\
\hline SHROOM3 & -1.46744 \\
\hline RBM11 & -1.47051 \\
\hline ARHGAP24 & -1.47717 \\
\hline GSTA5 & -1.48294 \\
\hline C1orf194 & -1.48387 \\
\hline DMD & -1.52499 \\
\hline RSPH1 & -1.52543 \\
\hline IGF1 & -1.54242 \\
\hline TMEM200A & -1.54474 \\
\hline PRRT4 & -1.55266 \\
\hline DLX4 & -1.55278 \\
\hline LOC645206 & -1.55438 \\
\hline FAM183A & -1.56155 \\
\hline LOC100128252 & -1.57934 \\
\hline TMEM56 & -1.58744 \\
\hline EFCAB1 & -1.60353 \\
\hline MURC & -1.62903 \\
\hline LOC400043 & -1.63097 \\
\hline DNAI2 & -1.63951 \\
\hline CXCR7 & -1.65679 \\
\hline ZNF702P & -1.66273 \\
\hline KCNAB1 & -1.70793 \\
\hline GBP7 & -1.72011 \\
\hline GABRE & -1.72196 \\
\hline CYP3A7 & -1.73123 \\
\hline CAMP & -1.83452 \\
\hline LEF1 & -1.85639 \\
\hline CD40LG & -1.87393 \\
\hline AOC3 & -1.88126 \\
\hline TCEA3 & -1.91778 \\
\hline PTGER3 & -2.00014 \\
\hline FAM125B & -2.01546 \\
\hline TCF7L1 & -2.03268 \\
\hline
\end{tabular}

Table S1 (continued)
Table S1 (continued)

\begin{tabular}{lc}
\hline id & $\log \mathrm{FC}$ \\
\hline ENPP3 & -2.05402 \\
CYP3A5 & -2.16341 \\
ITIH5 & -2.26283 \\
C8B & -2.31597 \\
NALCN & -2.56164 \\
\hline
\end{tabular}


Table S2 Detailed results of prognostic model using the multivariate Cox regression

\begin{tabular}{lccccc}
\hline id & coef & HR & HR.95L & HR.95H & pvalue \\
\hline CXCL14 & 0.197048 & 1.217802 & 1.003692 & 1.477588 & 0.045791 \\
SLC40A1 & 0.328027 & 1.388227 & 0.9675 & 1.991911 & 0.074976 \\
RNASE3 & 0.585181 & 1.795316 & 1.141325 & 2.824052 & 0.011344 \\
CCR3 & 0.280172 & 1.323357 & 1.006682 & 1.73965 & 0.044676 \\
RORA & -0.65037 & 0.521853 & 0.322487 & 0.844468 & 0.008089 \\
\hline
\end{tabular}

Table S3 The grouping information of IPF patients stratified by risk scores

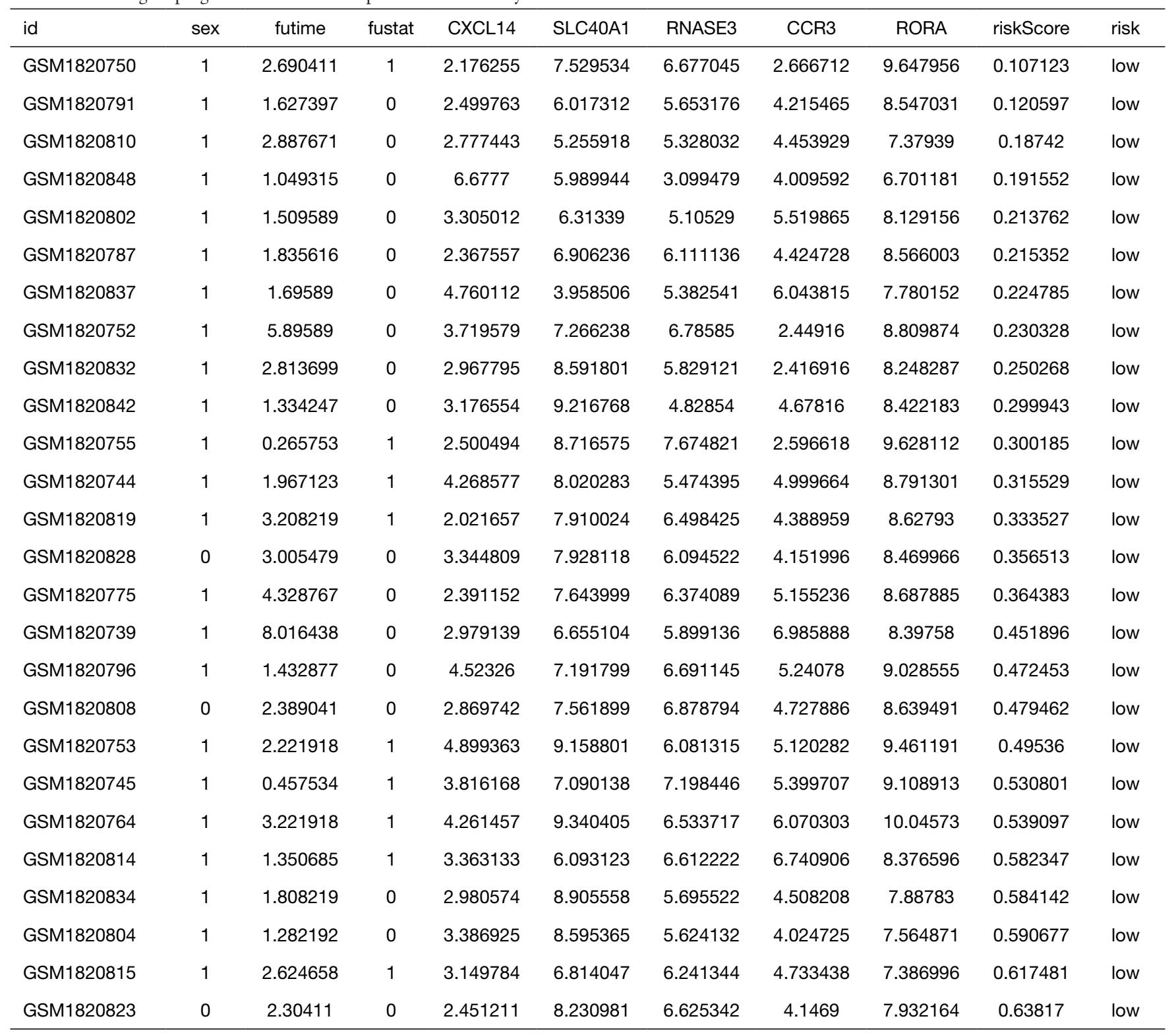

Table S3 (continued) 
Table S3 (continued)

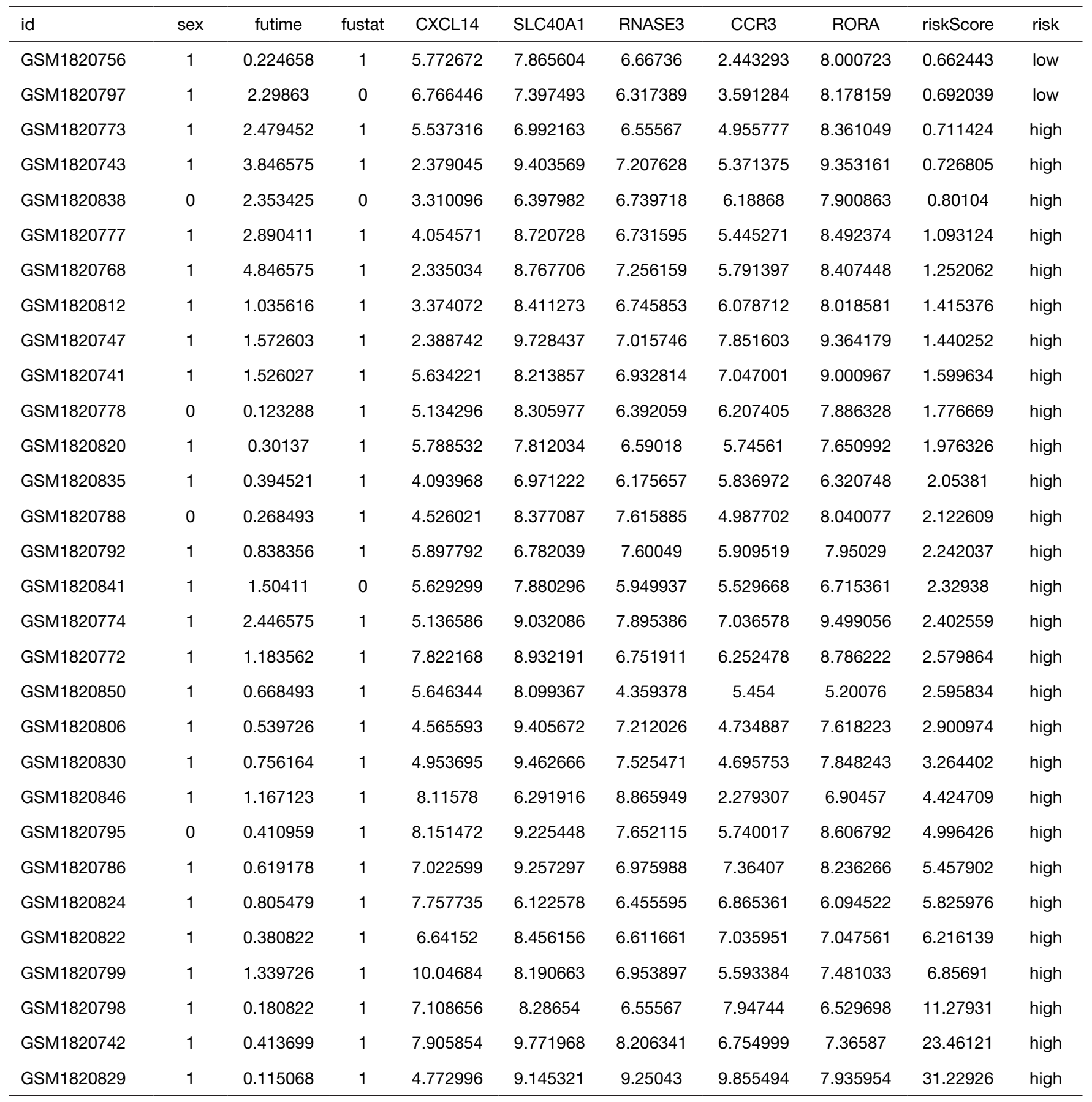

